\title{
A novel de-interlacing algorithm based on dynamic threshold motion detection
}

\author{
Yuan Ye \\ School of Business Information, Shanghai University of International Business and Economics, \\ Shanghai, 201620, China
}

email: aaaayuanye@126.com

Keywords: De-interlacing; Motion adaptive; Motion detection; Dynamic threshold

\begin{abstract}
In order to solve the noise and blur in traditional de-interlacing algorithm, a novel de-interlacing algorithm based on dynamic threshold motion detection is proposed to detect still and motion parts. First, the dynamic maximum and minimum are obtained according to different situations. Second, the motion detection information from five domain points in four fields are obtained. Then progressive image are obtained according to the motion detection information. Experimental results demonstrate that the algorithm can not only solve still image noise but also solve the motion blur. It can be used in the low-cost chip of digital video processing.
\end{abstract}

\section{Introduction}

De-interlacing is today a key technology in consumer TV that converts ordinary interlaced formats into progressive ones by reconstructing the missing lines. Some typical defects in video de-interlacing will cause uncomfortable visual artifacts and critical distortions in the output frames. In order to eliminate the defects, the de-interlacing technique has been widely and deeply studied in recent years.

The most common de-interlacing methods are grouped in three main categories: linear interpolation [1] ,motion adaptive methods [2][3][4] and motion compensated methods[5].

Linear interpolation include temporal (inter-field), spatial methods (intra-field). Temporal techniques work better than spatial techniques for static scenes, because when there is no motion, the missing lines are the same as the known previous ones. On the other hand, when there is motion in the scene, the previous lines contain information that is not coincident with the present data and ghosting, tearing or combing artifacts appear in the moving regions[5]. For these moving areas, spatial interpolation gives better results. Many spatial-temporal hybrid-de-interlacing techniques have been proposed to exploit the spatial and temporal correlation of video pictures and to overcome the artifacts. The corresponding techniques are called motion-adaptive algorithms. If the motion detection is not accurate, it will cause big noise or tearing or combing artifacts[4].

Motion compensated algorithms provide the highest reconstruction quality. They are computationally more expensive because they require the estimation of two-dimensional motion vectors and pixel shifting calculations. It can't be used in low-cost chip of digital video processing.

In this paper, a novel de-interlacing algorithm based on dynamic threshold motion detection is proposed. Five domain points in four fields are used to detect the motion situation of the interpolation point, according to the different situation the motion and static scenes can be correctly detected, and finally using the motion detection information progressive image are obtained. Experimental results demonstrate that the algorithm can get a good result.

\section{The Principle of De-interlacing}

The basic principle of de-interlacing is based on the temporal and spatial relativity of the sequences. Through de-interlacing, the missing lines can be reconstructed. Figure 1 describes the position of four fields data. The present missing point is in middle position of the third field. What we need to do is reconstruct this point. 

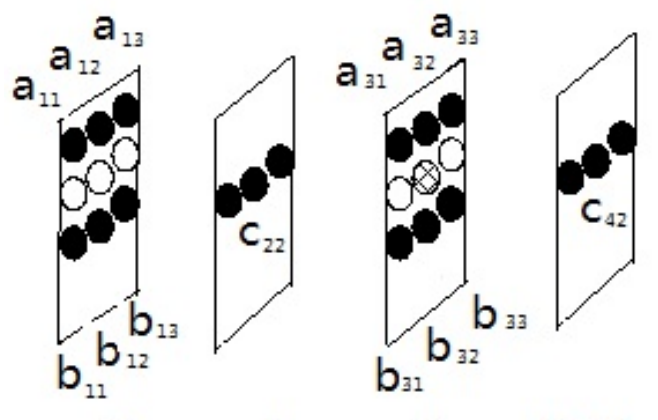

$$
n-2 \quad n-1 \quad n \quad n+1
$$

Fig.1 Space position illustration of interpolation point

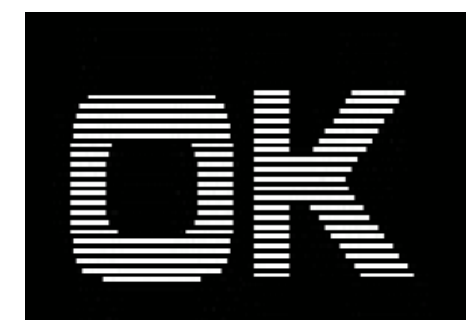

Fig.2 OK illustration

The principle of the traditional motion adaptive algorithm is that, if in the case of static scenes, the temporal filter is the best, while in the case of motion, spatial filter is the best. Through detecting the motion information automatically the purpose of motion adaptive is achieved.

\section{The Implementation of the De-interlacing Algorithm Based on Dynamic Threshold Motion Detection}

Obtain Maximum and Minimum Threshold Dynamically. There are many situations to determine the object is moved or not, a fixed threshold is not enough. As shown in Figure 2, the classical "OK" image in the test video, $\mathrm{O}$ is in the odd field, $\mathrm{K}$ is in the even field. If we want to reconstruct this missing point in the third field, four-field data is needed in this algorithm. We call this case as OESL (Odd Even Single Line), which belongs to a static scenes, in this case the threshold should be larger. The detection of OESL is as follows:

$$
\text { Er }=\left(a_{32}+b_{32}\right) / 2-c_{42}
$$

Where the positions of $a_{32} 、 b_{32} 、 c_{42}$ are shown in Figure 1, Er is the difference between the mean value of the upper and lower points and corresponding point of next field. If it is very large, it belongs to OESL.

$$
T_{\max }, T_{\min }=\left\{\begin{array}{rl}
T_{\max }=T_{1} T_{\min }=T_{2} & E r \geq T_{b} \\
T_{\max }=T_{1 s}+\frac{\left(E r-T_{m}\right)\left(T_{b}-T_{m}\right)}{T_{2}-T_{1 s}} T_{\min }=T_{1 s}+\frac{\left(E r-T_{m}\right)\left(T_{b}-T_{m}\right)}{T_{1}-T_{1 s}} & E r>T_{m} \\
T_{\max }=T_{1 s} T_{\min }=T_{1 s} & \text { else }
\end{array}\right.
$$

Here, $T_{1} 、 T_{2}$ are the maximum and minimum of OESL, the default value is 30 and $10 . T_{1 s}$ is threshold of normal situation, the default value is $4 ; T_{b}$ is big threshold of OESL, the default value is 200. $T_{m}$ is middle threshold of OESL, the default value is 70 . The formula shows that the maximum and minimum $T_{\min }, T_{\max }$ are variables according to different cases. If it belongs to OESL, the maximum and minimum are bigger. In order to prevent the images transition is not natural, a smooth transition is used when $\operatorname{Er}$ is between $\left[T_{m}, T_{b}\right]$.

\section{Obtain Motion Detection Information}

(1) Obtain maximum of five- point-four-field difference

$$
E=\max \left(E_{1}, E_{2}, E_{3}, E_{4}, E_{5}\right)
$$

Here, $E_{1}=\left|a_{32}-a_{12}\right|, E_{2}=\left|b_{32}-b_{12}\right|, E_{3}=\left|c_{42}-c_{22}\right|, E_{4}=\left|c_{41}-c_{21}\right|, E_{5}=\left|c_{43}-c_{23}\right|$. Interpolation point is shown in Figure 1, the grid point in the third field is present interpolation. $\mathrm{E}$ is the maximum of the five- point-four-field difference.

(2) Detect still point using five domain points

If the static condition is too strict, some static scenes can be misunderstood as a motion parts, and small noise can be seen in the video sequence. Therefore, still scenes will be detected further 
between five domain points in four fields, if there are 3 or more corresponding points are similar, it can be understood as a static scenes

$$
\begin{aligned}
& \text { if } E_{1}<T_{d} \text { or } E_{2}<T_{d} \text { or } E_{3}<T_{d} \text { or } E_{4}<T_{d} \text { or } E_{5}<T_{d} \\
& \text { still_sum }=\text { still_sum }+1 \\
& \text { end }
\end{aligned}
$$

Here, still _ sum indicates similar points number around the current point, its initial value is 0 , if three points or more in the five points are similar, it is considered that the present point is static. $T_{d}$ is the similarity threshold, which is set to 7 .

(3) Obtain motion detection information $I_{m}$

If $E r>T_{b}$, the point belongs to OESL, motion detection information $I_{m}$ can be obtained as follows:

$$
I_{m}= \begin{cases}0 & E \geq T_{\max } \\ 1 & E \leq T_{\min } \\ \frac{\mathrm{T}_{1}-E}{T_{1}-T_{2}} & \text { else }\end{cases}
$$

If $E r<=T_{m}$, the point belongs to normal situation, motion detection information $I_{m}$ can be obtained as follows:

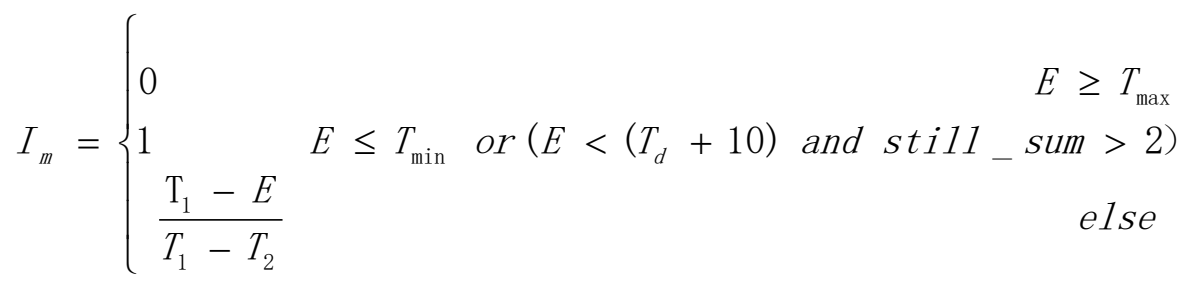

The formula shows that the static scenes can be detected further. Too strict static detection will result in noise. If there are more than three points around the point is judged to be static, it can be considered that the center point is still. At this point, $I_{m}$ should be corrected as 1 , which means it is still.

Intra Interpolation. When it is detected as moving scene, we can use the intra interpolation algorithm. In this paper, the intra interpolation algorithm is based on seven direction edge judgment [6][7], the edge direction can be determined by the relativity of image content, and edge of image can effectively protected using this interpolation algorithm.

Obtain Progressive Images. After motion detection information has been judged and intra interpolation are obtained, progressive images can be obtained using the following formula:

$$
F_{o}(x, y, n)= \begin{cases}F(x, y, n) & x \bmod 2=n \bmod 2 \\ \left(1-I_{m}\right) * F_{\text {intra }}(x, y, n)+I_{m} * F(x, y, n+1) & \text { else }\end{cases}
$$

Here, $F(x, y, n+1)$ represents the value of last field, $F_{o}(x, y, n)$ represents final output of the progressive image, $F_{\text {intra }}(x, y, n)$ represents intra interpolation. The formula shows that if $I_{m}=1$, it is static and next field can be used to improve the vertical resolution. if $I_{m}=0$, it is moving and intra interpolation can be used to avoid the motion blur caused by the inter interpolation.

\section{Simulation Results and Analysis}

This algorithm is simulated using MATLAB language. We choose the classic video image in special test disc. Fig.3 is the result of system simulation.. Fig.3(a) are original four fields video data; Fig. 3 (b) are the result of the traditional motion adaptive algorithm[4], we can see that "OK" is not perfect; Fig.3(c) are the result of de-interlacing algorithm based on dynamic threshold motion 
detection proposed here. Compared with 3 (b), the still scene "OK" and the moving pendulum are good. The image quality is improved. Fig.3 (d) is the details of Fig.3 (b) as well as the motion detection image of new algorithm. It can be seen that the moving parts and still parts are detected well, it can reduce the video stream 3D noise effectively.
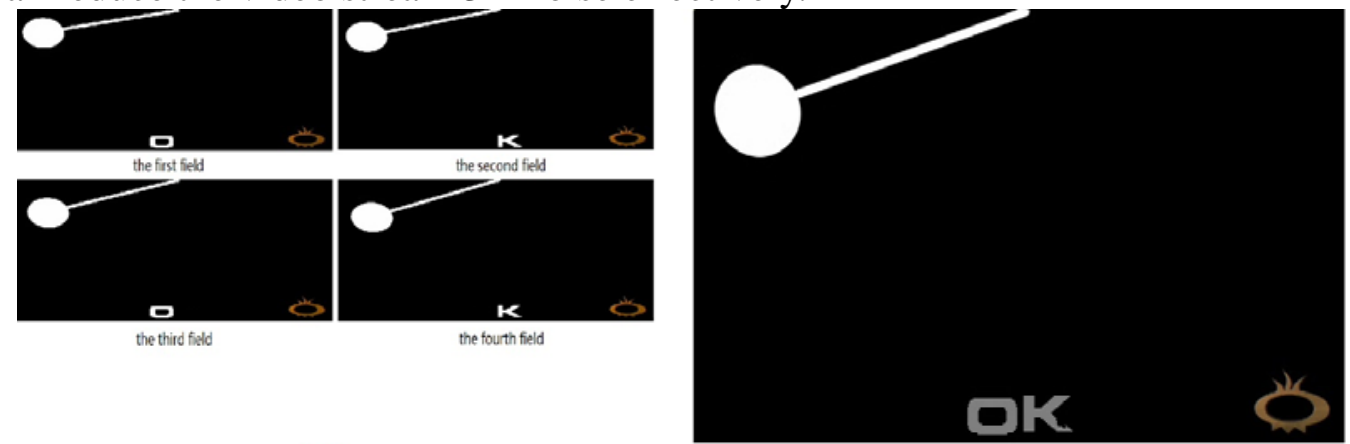

( a )

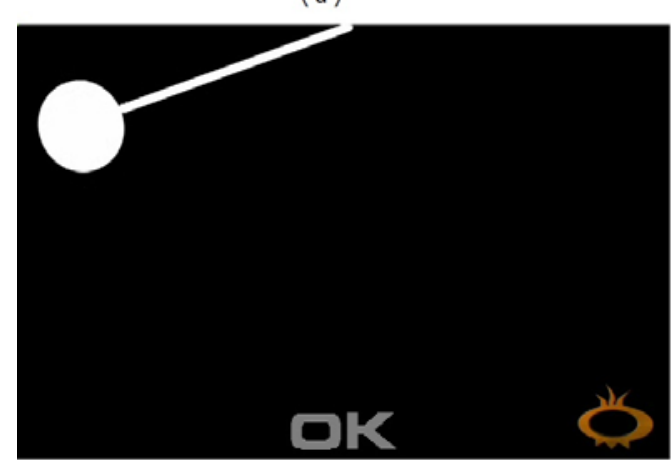

(b)

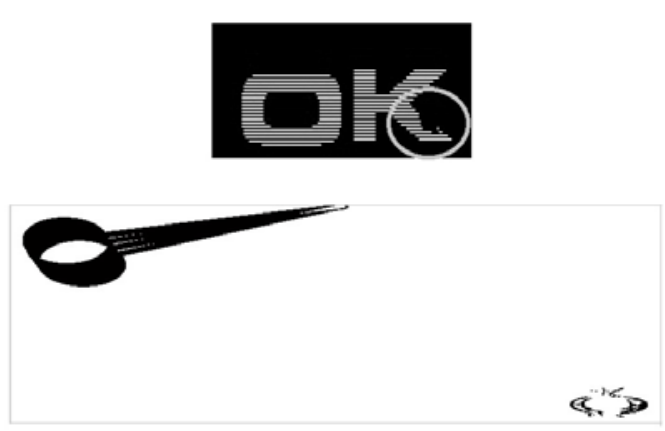

(d)
(a) Original results
(b)Traditional motion adaptive algorithm
(c) The new algorithm
(d) Detail enlargement and motion detection figure of the new algorithm

Fig.3 Simulation result
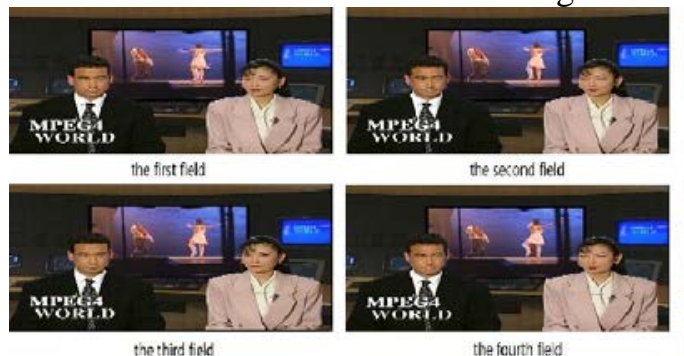

(a)
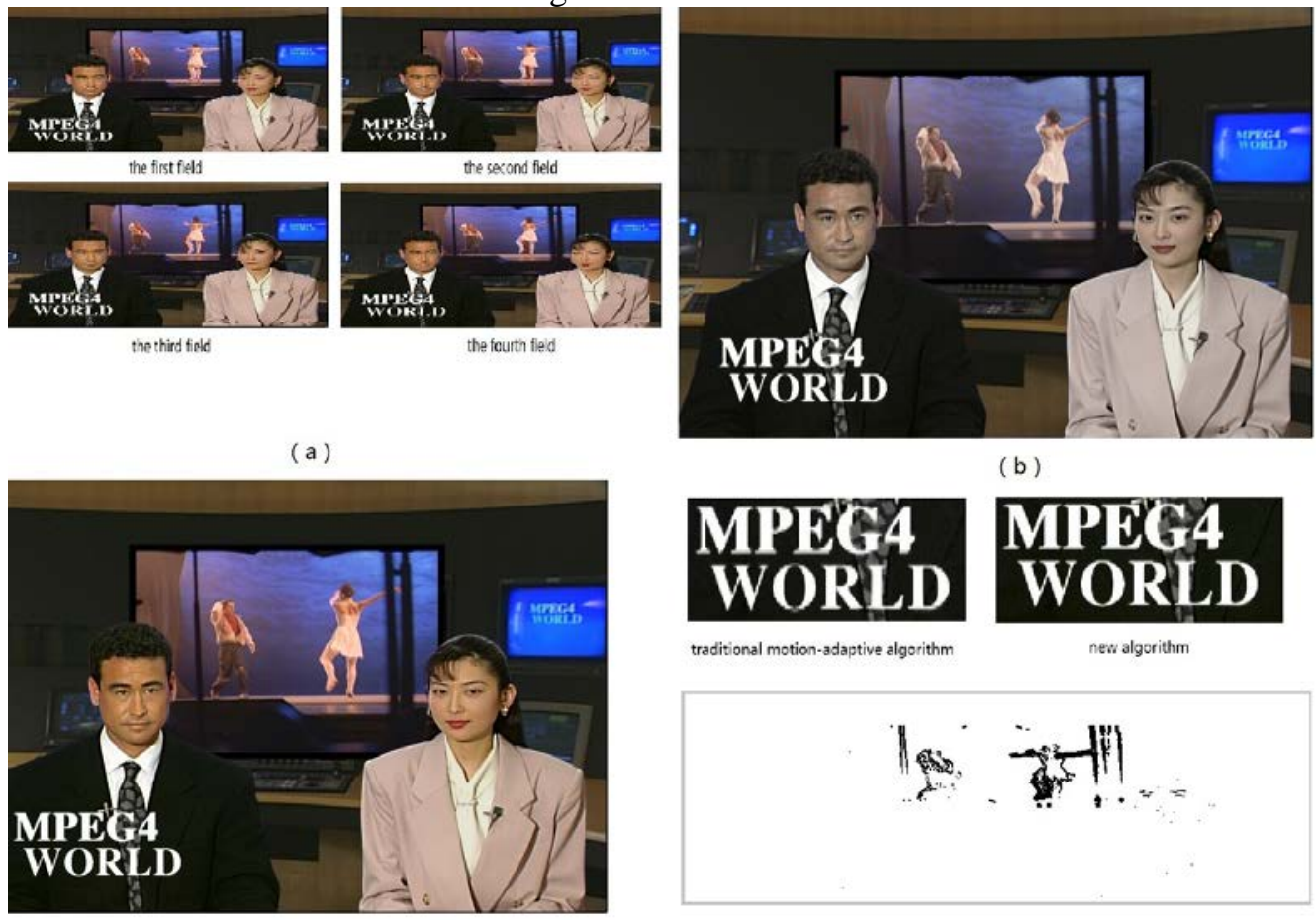

(b)

(c)

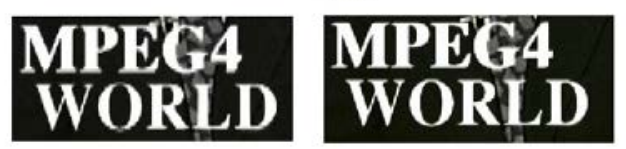

traditional motion-adaptive algorithm new algorithm

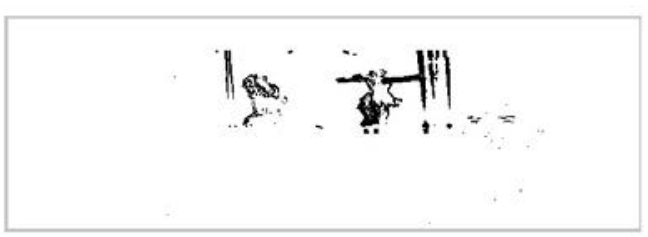

(d)
(a) Original results
(b)Traditional motion adaptive algorithm
(c)The new algorithm
(d) Detail enlargement and motion detection figure of the new algorithm Fig. 4 Simulation results.

Fig.4 (a) are the original four fields video data; Fig.4 (b) are the results of traditional motion adaptive algorithm, it can be seen in the picture that the vertical resolution of the text below is very 
low; Fig.4 (c) are the results of the new algorithm, and compared with Fig.4 (b), the rotating dancers don't blur and there are rich details in announcers and the text below. Fig. 4 (d) are the partial enlarged detail of fig.4(b).the text "O" is judged as moving part and intra interpolated, which will reduce vertical resolution. Fig.4(d) is a picture of the motion detection figure of new algorithm, it can be seen that the moving stage and dancers are detected very well. The experimental results show that good experimental results can be obtained using this novel de-interlacing algorithm.

\section{Conclusion}

A novel de-interlacing algorithm based on dynamic threshold motion detection is proposed to solve noise and blur phenomenon. First, dynamic maximum and minimum are obtained. Second, the motion detection information can be obtained through detection the relativity of five domain points in four fields, which can improve the accuracy of motion detection and avoid noise. Then progressive images are reconstructed according to the motion detection information. Experimental results show that the proposed algorithm can achieve the function of motion detection effectively. It can be used in the low-cost chip of digital video processing.

\section{References}

[1] Haan G de, Bellers E B. An De-interlacing overview [J].Proceedings of the IEEE, 1998, 86(9):1839-1857.

[2] LIU Zheng-lin, LIU Ju, ZOU Xue-cheng et al. A novel motion adaptive de-interlacing algorithm[J]. Microprocessors, 2008(6):116-118.

[3] Lin S F, ChangY L, Chen LG. Motion adaptive de-interlacing with horizontal motion detection and ELA with median [C]//Proceedings of 2003 IEEE International Symposium on Circuits and Systems(ISCAS 2003), 2003: 696-699.

[4] PU Yu-wei, YE Bing, ZENG De-rui, et al. A motion adaptive de-interlacing technique and VLSI architecture[J]. Video Engineering, 2011(21):40-44.

[5] YANG Liu, XU Wei-pu. A novel adaptive de-interlacing algorithm based on motion compensation[J]. Microelectronics and computer, 2002(9):4-6, 11.

[6] AN Hui-zhong 1 , LIU Wei-dong, A motion adaptive algorithm of de-interlacing based on improved edge-based line averaging[J], Electronic Design Engineering, 2014, 22 (5) , 187-189.

[7] YUAN Ye, TIAN Zhong-xu , New edge preserving enlargement algorithm for images[J], Journal of Computer Applications, 2012， 32(11) : 3182 -3184 